\title{
Statistical Wave Equation for Nonrelativistic Rigid Body Motions
}

\author{
George H. Goedecke \\ Physics Department, New Mexico State University, Las Cruces, NM, USA \\ Email: ggoedeck@nmsu.edu
}

How to cite this paper: Goedecke, G.H. (2017) Statistical Wave Equation for Nonrelativistic Rigid Body Motions. Journal of Modern Physics, 8, 1911-1932.

https://doi.org/10.4236/jmp.2017.812114

Received: October 9, 2017

Accepted: November 7, 2017

Published: November 10, 2017

Copyright $\odot 2017$ by author and Scientific Research Publishing Inc. This work is licensed under the Creative Commons Attribution International License (CC BY 4.0).

http://creativecommons.org/licenses/by/4.0/

\begin{abstract}
In this work, the general nonrelativistic classical statistical theory presented in an earlier paper (J. Mod. Phys. 8, 786 (2017)) is applied in detail to the Euler angle and center-of-mass coordinates of an extended rigid body with arbitrary distributions of mass and electric charge. Results include the following: 1) The statistical theory spin angular momentum operators are independent of the body's morphology; 2) These operators obey the usual quantum commutation rules in a non-rotating center-of-mass (CM) reference frame, but left-handed rules in a rotating body-fixed CM frame; 3 ) Physical boundary conditions on the Euler angle wavefunctions restrict all mixed spin wavefunctions to a superposition of half-odd-integer spin eigenstates only, or integer spin eigenstates only; 4) Spin $s$ eigenfunctions are also Hamiltonian eigenfuctions only if at least two of the body's principal moments of inertia are equal; 5) For a spin $s$ body with nonzero charge density in a magnetic field, the theory automatically yields $2 s+1$ coupled wave equations, valid for any gyromagnetic ratio; and 6) For spin 1/2 the two coupled equations become a Pauli-Schrödinger equation, with the Pauli matrices appearing automatically in the interaction Hamiltonian.
\end{abstract}

\section{Keywords}

Stochastic Classical Mechanics, Stochastic Quantum Mechanics, Stochastic Spin

\section{Introduction}

In an earlier paper [1], hereafter referred to as [I], we proved that any possible statistical treatment of all possible nonrelativistic classical motions of any Lagrangian system that is acted upon by a stochastic force field always yields the corresponding Schrödinger equation, with its usual boundary conditions and 
solutions, as an essential statistical equation that must be obeyed by the system, once an unknown constant is set equal to $\hbar$. We derived the conventional canonical quantization rule between each coordinate and its conjugate momentum operator, and also the conventional prescription for the Hamiltonian operator, namely, that it must be the classical Hamiltonian in the $\mathrm{N}$-dimensional metric configuration space specified by the classical kinetic energy of the system, with the conjugate momentum $N$-vector replaced by $-i \hbar$ times the vector gradient operator in that space. These results imply that a classical statistical analog of the quantum spin operator should exist for all nonrelativistic rigid rotations of any extended model particle.

Many authors have considered classical spinning top models and their possible connections to quantum spin and magnetic moment; we refer to a few examples that seem important in regard to this work [2]-[12]. These treatments either postulate the usual commutation rules for the Cartesian components of the spin operator in the non-rotating coordinate system by analogy with the rules for orbital angular momentum, or they begin with an Euler angle description of rigid rotations, and simply postulate that the momenta conjugate to the angles become operators given by $-i \hbar$ times derivatives with respect to the angles, in analogy with conjugate translational momenta. As discussed below, our statistical treatment does not require any such postulates.

There are two well-known objections to nonrelativistic spinning top models. One is that tangential speeds involved in any rigidly rotating object with mass and effective radius as small as those of an electron must far exceed the vacuum speed of light in order that the model have spin angular momentum of order $\hbar$ [13]. The other objection is that if the spin eigenfunctions must be single-valued periodic functions of the Euler angles with period $2 \pi$, then half-odd-integer spin is not allowed. These objections were addressed and at least partially resolved in [I], where we concluded that rotations and translations of electrons and other particles having extremely small mass and size must always be treated relativistically, while larger particles often may be treated non-relativistically. We also showed that half-odd-integer spin is allowed. (Some further rationale for half-odd-integer spin is provided in the paragraphs following Equation (52) below.)

This paper concentrates on applying the general classical statistical theory (CST) derived in [I] to a nonrelativistically rotating and translating massive rigid body having arbitrary internal structure and containing a co-moving electric charge distribution. The body is immersed in a stochastic force field, a specified magnetic field, and perhaps other specified fields. In [I], only a spherically symmetric body immersed in a stochastic force field but not in magnetic or other specified fields was treated. Following several authors [2] [5] [6], a rigid spherically symmetric extended particle model is used for simplicity in the text below to represent a magnetic dipole; Appendix A provides a new treatment of a rigid extended particle magnetic dipole model having arbitrary structure. 
Section 2 defines center-of-mass (CM) and Euler angle coordinates and obtains a new general tensor calculus representation of the nonrelativistic classical Lagrangian, Hamiltonian, and rotational motion equations in terms of the metric in the Euler angle 3-space. Section 3 utilizes these new results and other results obtained in [I] to derive the statistical theory spin and Hamiltonian operators and thus the general statistical Schrödinger equation for the system. The Cartesian components of the spin angular momentum operator in both the non-rotating and rotating CM frames of reference are linear combinations of derivatives with respect to the Euler angles; they are actually derived from the theory, not just postulated or inferred by analogy. While the non-rotating frame components satisfy the usual commutation rules for spin operators, the body-fixed frame components satisfy left-handed commutation rules. A new derivation of the simultaneous eigenfunctions of the square of the spin operator and its rotating and non-rotating frame $z$-components is provided. For example, for spin $1 / 2$, this nonrelativistic theory automatically yields a modified Pauli-Schrödinger equation involving the Pauli spin matrices and a two-element spinor wavefunction. The new modified equation contains a rigid rotator term and a term involving the square of the magnetic field, as well as an interaction Hamiltonian having the usual form but valid for any magnetogyric ratio. Application of physical boundary conditions with respect to the Euler angles provides a new derivation of known behavior, namely, that a general wavefunction must be a superposition of half-odd-integer spin eigenstates only, or integer spin eigenstates only. Section 4 provides a summary and discussion of the results of this work, including a comparison of orbital and spin angular momentum in rotator models. Section 5 considers conclusions that may and may not be drawn from this work. Appendix A provides a new derivation of the Lagrangian and Hamiltonian for a rigid charged rotator of arbitrary morphology in an electromagnetic field, as well as consequences for a rotator having cylindrical but not spherical symmetry. This appendix also provides a new derivation of the fact that the Euler angle spin angular momentum operators are independent of the structure of the model rotator, whereby any object in a nonrelativistic rigid rotator spin eigenmode must have either odd-half-integer or integer spin.

\section{Classical Nonrelativistic Rotator}

\subsection{Euler Angles and Angular Velocity}

Some of what follows in this section is treated in textbooks and/or in [I], but we present it here for clarity and to establish notation. We use a conventional choice of Euler angle coordinates, the "zyz" set [14] used in several textbooks on mathematical methods of physics, e.g., Arfken [15]. We designate these coordinates $\alpha^{b}$, where indices $b, c, d, \cdots$ from the first part of the alphabet range and sum from 1 to 3 . With this set of angles, a general transformation of Cartesian coordinates from a non-rotating system with Cartesian unit basis vectors $\hat{\boldsymbol{e}}_{i}$ to a rotating system with Cartesian unit basis vectors $\hat{\overline{\boldsymbol{e}}}_{i}$, with the 
origin of both systems at the body CM, is obtained by specifying right-hand-screw rotations about the non-rotating system $z$-axis by angle $\alpha^{1}$, then about the new $y$-axis by angle $\alpha^{2}$, then finally about the new $z$-axis by angle $\alpha^{3}$. In general, the ranges of the angles should be $(-\infty, \infty)$, because the body may just keep on rotating about any axis. However, physical objects should look the same modulo $2 \pi$ in all angles, so observable functions such as coordinate probability densities should be periodic in each Euler angle with period $2 \pi$. The Cartesian basis vectors in the two systems are related by

$$
\hat{\boldsymbol{e}_{i}}=R_{i j}^{z}\left(\alpha^{3}\right) R_{j k}^{y}\left(\alpha^{2}\right) R_{k l}^{z}\left(\alpha^{1}\right) \hat{\boldsymbol{e}}_{l}=R_{i l}(\alpha) \hat{\boldsymbol{e}}_{l},
$$

where the matrices $R^{z}$ and $R^{y}$ are given by

$$
R^{z}(\mu)=\left(\begin{array}{ccc}
\cos \mu & \sin \mu & 0 \\
-\sin \mu & \cos \mu & 0 \\
0 & 0 & 1
\end{array}\right) ; \quad R^{y}(\mu)=\left(\begin{array}{ccc}
\cos \mu & 0 & -\sin \mu \\
0 & 1 & 0 \\
\sin \mu & 0 & \cos \mu
\end{array}\right),
$$

where here $\mu$ is a dummy variable. Thus, the complete rotation is specified by the matrix $R(\alpha)=R^{z}\left(\alpha^{3}\right) R^{y}\left(\alpha^{2}\right) R^{z}\left(\alpha^{1}\right)$, which is orthogonal with determinant +1 (as are the constituent matrices). The set of all such $3 \times 3$ matrices forms the defining irreducible representation of the rotation group SO(3)

The angular velocity 3 -vector can be found from the relations defining rigidly rotating Cartesian coordinates,

$$
\mathrm{d} \hat{\overline{\boldsymbol{e}_{i}}} / \mathrm{d} t=\boldsymbol{\omega} \times \hat{\overline{\boldsymbol{e}_{i}}}
$$

where $\boldsymbol{\omega}$ is the instantaneous angular velocity, and $\times$ here indicates the cross-product. The Cartesian components $\omega_{i}=\hat{\boldsymbol{e}}_{i} \cdot \boldsymbol{\omega}$ in the non-rotating frame, and $\bar{\omega}_{i}=\hat{\overline{\boldsymbol{e}}}_{i} \cdot \boldsymbol{\omega}$ in the rotating frame, can be obtained using Equations (1)-(3). The results are

$$
\omega_{i}=a_{i b} \dot{\alpha}^{b} ; \quad \bar{\omega}_{i}=b_{i b} \dot{\alpha}^{b}=R_{i k} \omega_{k},
$$

where we have specified the time-dependent trajectories of the Euler angles by $\alpha^{b}=\alpha^{b}(t)$. The matrices $(a)$ and $(b)$ are given by

$$
(a)=\left(\begin{array}{ccc}
0 & -\sin \alpha^{1} & \sin \alpha^{2} \cos \alpha^{1} \\
0 & \cos \alpha^{1} & \sin \alpha^{2} \sin \alpha^{1} \\
1 & 0 & \cos \alpha^{2}
\end{array}\right) ; \quad(b)=\left(\begin{array}{ccc}
-\sin \alpha^{2} \cos \alpha^{3} & \sin \alpha^{3} & 0 \\
\sin \alpha^{2} \sin \alpha^{3} & \cos \alpha^{3} & 0 \\
\cos \alpha^{2} & 0 & 1
\end{array}\right) \text {. }
$$

Note that Equation (4) implies $(b)=(R)(a)$.

\subsection{Classical Nonrelativistic Rotator Dynamics}

As mentioned above, we first consider a very simple model rotator, a rigid extended spherically symmetric object having only the attributes of an electric charge distribution with total charge $q$ which may be nonzero or zero, a mass distribution with mass $m>0$, a principal moment of inertia $I$ about the CM, and the 3-vectors CM position $\boldsymbol{X}(t)$, intrinsic magnetic dipole moment $\boldsymbol{\mu}(t)$, and angular velocity $\omega(t)$, both of the latter about the CM. (In Appendix A, 
we consider a rigid object of arbitrary morphology in a magnetic field, which we did not treat in [I].) We neglect gravitational interactions. The classical definition of intrinsic magnetic moment (Gaussian units) is

$$
\boldsymbol{\mu}(t)=(2 c)^{-1} \int \mathrm{d}^{3} x^{\prime} \boldsymbol{x}^{\prime} \times \boldsymbol{J}(\boldsymbol{x}, t),
$$

where $\boldsymbol{x}^{\prime}=\boldsymbol{x}-\boldsymbol{X}(t)$, and $\boldsymbol{J}(\boldsymbol{x}, t)$ is the electric current density. For a spherically symmetric translating and rigidly rotating model,

$$
\boldsymbol{J}(\boldsymbol{x}, t)=q\left[\dot{\boldsymbol{X}}(t)+\boldsymbol{\omega}(t) \times \boldsymbol{x}^{\prime}\right] f_{q}\left(x^{\prime}\right),
$$

where $x^{\prime}=\left|x^{\prime}\right|$, and the electric charge density is $q f_{q}\left(x^{\prime}\right)$, so that $\int \mathrm{d}^{3} x^{\prime} f_{q}\left(x^{\prime}\right)=1$. Combining these equations and noting that $\int \mathrm{d}^{3} x^{\prime} \boldsymbol{x}^{\prime} f_{q}\left(x^{\prime}\right)=0$ yields

$$
\boldsymbol{\mu}(t)=q(2 c)^{-1}\left[\frac{2}{3} \int \mathrm{d}^{3} x^{\prime} x^{\prime 2} f_{q}\left(x^{\prime}\right)\right] \boldsymbol{\omega}(t) .
$$

The nonrelativistic kinetic spin angular momentum is

$$
\boldsymbol{S}^{K}(t)=m \int \mathrm{d}^{3} x^{\prime}\left[\boldsymbol{x}^{\prime} \times\left(\boldsymbol{\omega}(t) \times \boldsymbol{x}^{\prime}\right)\right] f_{m}\left(x^{\prime}\right)=I \boldsymbol{\omega}(t),
$$

where $m f_{m}\left(x^{\prime}\right)$ is the mass density, so that $\int \mathrm{d}^{3} x^{\prime} f_{m}\left(x^{\prime}\right)=1$, and the moment of inertia $I$ is given by

$$
I=\frac{2}{3} m \int \mathrm{d}^{3} x^{\prime} x^{\prime 2} f_{m}\left(x^{\prime}\right) .
$$

Combining Equations (8)-(10) yields

$$
\boldsymbol{\mu}(t)=(g q / 2 m c) S^{K}=\tilde{g} I \omega(t),
$$

where the dimensionless parameter $g$ is defined by

$$
g=\int \mathrm{d}^{3} x^{\prime} x^{\prime 2} f_{q}\left(x^{\prime}\right) / \int \mathrm{d}^{3} x^{\prime} x^{\prime 2} f_{m}\left(x^{\prime}\right),
$$

and the magnetogyric ratio $\tilde{g}$ is defined by

$$
\tilde{g}=g q / 2 m c \text {. }
$$

We provided the detailed derivation above not only for clarity but also to emphasize that the intrinsic magnetic moment of a spherically symmetric rigidly rotating charged body is proportional to the kinetic spin angular momentum $\boldsymbol{S}^{K}$, not to the spin angular momentum $\boldsymbol{S}$ to be defined below that is canonically conjugate to the Euler angles. This point has been emphasized by several authors [6] [11] [12].

The original Pauli-Schrödinger treatment and the Dirac equation yield $g=2$ for a bare electron. For nonrelativistic spherical rotators with $f_{q}=f_{m}$, i.e. with charge density proportional to mass density, Equation (12) clearly yields $g=1$. It may be interesting that a circular disk geometry with charge uniformly distributed around the circumference and mass uniformly distributed in the disk yields exactly $g=2$. This result can be derived easily from the analogs of Equation (12) for nonspherical objects, as shown in Appendix A.

The conventional nonrelativistic Lagrangian for the charged translating rigid 
rotator system considered here can be derived quite easily as the sum of the translational kinetic energy (KE) of the $\mathrm{CM}$, the rotational $\mathrm{KE}$ about the $\mathrm{CM}$, and the interaction Lagrangian

$$
L_{\text {int }}=\int \mathrm{d}^{3} x\left(-\rho_{q} \varphi+c^{-1} \boldsymbol{J} \cdot \boldsymbol{A}\right)
$$

for the interaction of external electromagnetic potentials $(\varphi, \boldsymbol{A})$ with any electrical charge-current densities $\left(\rho_{q}, \boldsymbol{J}\right)$. For the model particle used here, sharply localized around $\boldsymbol{x}=\boldsymbol{X}(t)$, the appropriate approximation for the interaction Lagrangian is obtained by a Taylor expansion of the external fields about the CM and use of Equation (7) for $\boldsymbol{J}$. The resulting expression for $L$ that is valid through dipole moment interactions is

$$
L=\left[\frac{1}{2} m \tilde{\boldsymbol{V}}^{2}-q \varphi+c^{-1} q \boldsymbol{A} \cdot \tilde{\boldsymbol{V}}\right]+\left[\frac{1}{2} I \boldsymbol{\omega}^{2}+\tilde{g} I \boldsymbol{\omega} \cdot \boldsymbol{B}\right],
$$

where $\tilde{\boldsymbol{V}}=\hat{\boldsymbol{e}}_{i} \dot{X}^{i}$ is the 3 -vector CM velocity, and the fields $\varphi, \boldsymbol{A}$ and the magnetic field (flux density) $\boldsymbol{B}$ are evaluated at the CM. Clearly, the first bracket is $L_{t r}$, the Lagrangian involving the translational motion of the CM, and the second bracket is $L_{r o t}$, the Lagrangian involving the rotational motion, including the usual interaction $\boldsymbol{\mu} \cdot \boldsymbol{B}$ of a magnetic dipole moment with a magnetic field. Of course, if the magnetic field is anything other than a constant vector, this rotational interaction term influences the translational motion as well, as will be discussed below. Note that the electric dipole moment about the $\mathrm{CM}$ is zero for this model extended particle, since the CM is also the center of charge.

Using the general boldface notation developed in [I], the Hamiltonian can be obtained without specifying the rotational coordinates. First, define the conjugate momentum 3-vectors

$$
\begin{gathered}
\tilde{\boldsymbol{P}}=\partial L / \partial \tilde{\boldsymbol{V}}=m \tilde{\boldsymbol{V}}+q \boldsymbol{A} / c ; \\
\boldsymbol{S}=\partial L / \partial \boldsymbol{\omega}=I \boldsymbol{\omega}+I \tilde{g} \boldsymbol{B},
\end{gathered}
$$

where $\tilde{\boldsymbol{P}}$ is the translational momentum conjugate to the CM velocity $\tilde{\boldsymbol{V}}$, and $\boldsymbol{S}$ is the intrinsic (spin) angular momentum about the CM, conjugate to $\boldsymbol{\omega}$. Then the Hamiltonian is given as usual by $H=\tilde{\boldsymbol{V}} \cdot \tilde{\boldsymbol{P}}+\boldsymbol{\omega} \cdot \boldsymbol{S}-L$. Applying Equations (14)-(16) yields

$$
H=\frac{1}{2 m}(\tilde{\boldsymbol{P}}-q \boldsymbol{A} / c)^{2}+\frac{1}{2 I}(\boldsymbol{S}-I \tilde{g} \boldsymbol{B})^{2}+q \varphi .
$$

This Hamiltonian is clearly equal to the sum of the kinetic and potential energies. It is conserved if neither of the potentials $\boldsymbol{A}$ and $\varphi$ depend explicitly on the time. Note that the effective interaction Hamiltonian involving the spin is the cross-term in the second term, $-\tilde{g} \boldsymbol{B} \cdot \boldsymbol{S}$, which is generally misinterpreted as $\boldsymbol{-} \boldsymbol{\mu} \cdot \boldsymbol{B}$. This form of the classical Hamiltonian for a system of one extended spherically symmetric rotating charged particle in electromagnetic fields was presented e.g. by Young [6], but has not been included in standard textbooks, despite the fact that once the angular velocity is expressed in terms of a set of 
Euler angles, Hamilton's canonical equations yield the correct Euler-Lagrange equations of motion for the rotation only if the second term in Equation (17) is present in its entirety.

At this point we should note that the treatment above also accomodates rigidly rotating objects that possess a magnetic moment and nonzero charge density but zero total electric charge. For such objects, one may put $q=0$ in the Hamiltonian (17), and simply choose a nonzero value of $g$ and a nonzero reference value such as $q=e$ in the definition of $\tilde{g}$, Equation (13).

Now, using Equation (4), we express the rotational kinetic energy $T_{\text {rot }}$ in terms of the Euler angles:

$$
\begin{gathered}
T_{\text {rot }}=\frac{1}{2} I \omega_{i} \omega_{i}=\frac{1}{2} m\left[\frac{I}{m} a_{i b} a_{i c} \dot{\alpha}^{b} \dot{\alpha}^{c}\right] ; \\
T_{\text {rot }}=\frac{1}{2} I \bar{\omega}_{i} \bar{\omega}_{i}=\frac{1}{2} m\left[\frac{I}{m} b_{i b} b_{i c} \dot{\alpha}^{b} \dot{\alpha}^{c}\right] .
\end{gathered}
$$

These expressions immediately reveal the covariant metric $g_{b c}^{r o t}=\frac{I}{m} a_{i b} a_{i c}=\frac{I}{m} b_{i b} b_{i c}$ in the Euler angle 3-space. Since $g_{b c}^{\text {rot }}=\boldsymbol{e}_{b} \cdot \boldsymbol{e}_{c}$ in terms of the covariant (subscripted) coordinate basis vectors in that space, and $g_{\text {rot }}^{b c}=\boldsymbol{e}^{b} \cdot \boldsymbol{e}^{c}$ in terms of the contravatiant (superscripted) basis vectors, and also $\delta_{b}^{c}=\boldsymbol{e}_{b} \cdot \boldsymbol{e}^{c}$, these basis vectors must satisfy

$$
\begin{gathered}
\boldsymbol{e}_{b}=\sqrt{\frac{I}{m}} a_{i b} \hat{\boldsymbol{e}}_{i}=\sqrt{\frac{I}{m}} b_{i b} \hat{\overline{\boldsymbol{e}}}_{i} ; \\
\boldsymbol{e}^{c}=\sqrt{\frac{m}{I}} a_{c j}^{-1} \hat{\boldsymbol{e}}_{j}=\sqrt{\frac{m}{I}} b_{c j}^{-1} \hat{\overline{\boldsymbol{e}}}_{j} ;
\end{gathered}
$$

These relations imply that $g_{\text {rot }}^{c d}=\frac{m}{I} a_{c j}^{-1} a_{d j}^{-1}$, which in turn implies $g_{b c}^{\text {rot }} g_{\text {rot }}^{c d}=\delta_{b}^{d}$, which must be the case. Also, it is straightforward to show from Equations (20) and (21) that the affine connections $\Gamma_{b c}^{d}$, which are defined by the relation

$$
\partial_{b} \boldsymbol{e}_{c}=\Gamma_{b c}^{d} \boldsymbol{e}_{d},
$$

are not symmetric under interchange of their lower indices. Therefore, the Euler angle space is a space with torsion, and the affine connections are not equal to the corresponding Christoffel symbols, as they are in a torsion-free space. However, the connections may all be evaluated using Equation (22) and Equations (20) and (21) and their inverses. We will need only one of the connections in this paper, which we derive below. (A feature of Euler angle metric 3-spaces that is interesting in its own right is that the Riemann-Christoffel curvature tensor, given by $R_{c a b}^{d}=\partial_{a} \Gamma_{b c}^{d}-\partial_{b} \Gamma_{a c}^{d}+\Gamma_{a c}^{d} \Gamma_{b c}^{e}-\Gamma_{b e}^{d} \Gamma_{a c}^{e}$, is zero, so the space is a flat space with torsion.) Note that our use of the coordinate basis vectors and the definition of the affine connection given above correspond to the usages in [I] and in the textbooks by Lichnerowicz [16] and Hartle [17].

The $3 \times 3$ matrix of the covariant components of the metric is given by $g^{\text {rot }}=\frac{I}{m}(a)^{\mathrm{T}}(a)=\frac{I}{m}(b)^{\mathrm{T}}(b) ;$ both expressions yield 


$$
\left(g^{\text {rot }}\right)=\frac{I}{m}\left(\begin{array}{ccc}
1 & 0 & \cos \alpha^{2} \\
0 & 1 & 0 \\
\cos \alpha^{2} & 0 & 1
\end{array}\right)
$$

Each covariant metric component has physical dimension (length) ${ }^{2}$. We may show fairly easily from Equations (5) and (21) that

$$
\partial_{b} \boldsymbol{e}^{b}=\left(\partial_{b} a_{b j}^{-1}\right) a_{j c} \boldsymbol{e}^{c}=-\Gamma_{b c}^{b} \boldsymbol{e}^{c}=-\left(\cot \alpha^{2}\right) \boldsymbol{e}^{2}
$$

Also, from Equation (23), $\sqrt{|g .|}=(I / m)^{3 / 2} \sin \alpha^{2}$, so that

$$
\boldsymbol{e}^{c} \partial_{c} \ln \sqrt{|g .|}=\left(\cot \alpha^{2}\right) \boldsymbol{e}^{2}
$$

Therefore, the general torsion-free $\mathrm{N}$-space identity

$$
\int \mathrm{d}^{N} x \sqrt{|g \cdot|} \nabla f=0
$$

is still valid for 3-dimensional Euler angle subspaces, despite their nonzero torsion. (In Equation (24), the integration extends over all $\mathrm{N}$-space, and $f$ is any function of the coordinates $x^{1}, x^{2}, x^{3}, \cdots, x^{N}$ that satisfies periodic boundary conditions.) This identity is necessary in establishing the Hermitean character of the Euler angle spin operators.

Writing out $T_{\text {rot }}$ yields

$$
T_{\text {rot }}=\frac{1}{2} I\left[\left(\dot{\alpha}^{1}\right)^{2}+\left(\dot{\alpha}^{3}\right)^{2}+2 \dot{\alpha}^{1} \dot{\alpha}^{3} \cos \alpha^{2}+\left(\dot{\alpha}^{2}\right)^{2}\right] .
$$

The magnetic interaction term in the Lagrangian is then

$$
L_{\text {int }}^{\text {mag }}=\tilde{g} I \boldsymbol{\omega} \cdot \boldsymbol{B}=\tilde{g} I B_{i} a_{i b} \dot{\alpha}^{b}=\tilde{g} I \bar{B}_{i} b_{i b} \dot{\alpha}^{b},
$$

where here the $\bar{B}_{i}$ are defined by $\bar{B}_{i}=R_{i k} B_{k}$, as if they were the barred (rotating) frame Cartesian components of an ordinary 3-vector. Note that the $\bar{B}_{i}$ depend on the Euler angles and thus are time-dependent even if the $B_{i}$ are not. Thus, the rotational part of the Lagrangian (14) may be expressed as

$$
L_{\text {rot }}=\frac{1}{2} m g_{b c}^{r o t} \dot{\alpha}^{b} \dot{\alpha}^{c}+\tilde{g} I B_{i} a_{i b} \dot{\alpha}^{b}=\frac{1}{2} m g_{b c}^{r o t} \dot{\alpha}^{b} \dot{\alpha}^{c}+\tilde{g} I \bar{B}_{i} b_{i b} \dot{\alpha}^{b} .
$$

The momenta conjugate to the angles are

$$
P_{b}=\partial L / \partial \dot{\alpha}^{b}=I a_{i b}\left(a_{i c} \dot{\alpha}^{c}+\tilde{g} B_{i}\right)=I b_{i b}\left(b_{i c} \dot{\alpha}^{c}+\tilde{g} \bar{B}_{i}\right) .
$$

These momenta have the dimension of angular momentum. Contraction with $a_{b k}^{-1}$ and $b_{b k}^{-1}$ and comparison with Equation (16) yields

$$
S_{k}=a_{b k}^{-1} P_{b}=I\left(\omega_{k}+\tilde{g} B_{k}\right) ; \quad \bar{S}_{k}=b_{b k}^{-1} P_{b}=I\left(\bar{\omega}_{k}+\tilde{g} \bar{B}_{k}\right),
$$

where the $\left(S_{k}, \bar{S}_{k}\right)$ are the (non-rotating, rotating) Cartesian components of the conjugate spin angular momentum 3-vector, respectively. Then, from the definition of the rotational part of the Hamiltonian, $H_{\text {rot }}=\dot{\alpha}^{b} P_{b}-L_{\text {rot }}$, where $L_{\text {rot }}$ is given by Equation (27), one easily obtains the rotational part of Equation (17), $H_{\text {rot }}=\frac{1}{2 I}(\boldsymbol{S}-I \tilde{g} \boldsymbol{B})^{2}$. Altogether, the classical Lagrangian and Hamiltonian in terms of Cartesian CM coordinates and the three Euler angle 
coordinates are given by

$$
\begin{aligned}
& L=\left[\frac{1}{2} m \delta_{i j} \dot{X}^{i} \dot{X}^{j}-q \varphi+q c^{-1} \dot{X}^{i} A_{i}\right]+\left[\frac{1}{2} m g_{b c}^{r o t} \dot{\alpha}^{b} \dot{\alpha}^{c}+\tilde{g} I B_{i} a_{i b} \dot{\alpha}^{b}\right], \\
& H=\frac{1}{2 m}\left(\tilde{P}_{i}-q c^{-1} A_{i}\right)\left(\tilde{P}_{i}-q c^{-1} A_{i}\right)+q \varphi+\frac{1}{2 I}\left(S_{i}-I \tilde{g} B_{i}\right)\left(S_{i}-I \tilde{g} B_{i}\right),
\end{aligned}
$$

where we have used the non-rotating frame components $S_{i}=a_{b i}^{-1} P_{b}$ and $B_{i}$ since the latter are presumed known and may be constants, as mentioned above.

The classical motion equations are derived from Equations (30) and (31) directly. After some algebra, the Euler-Lagrange (EL) equations applied to Equation (30) yield the following 3-vector classical motion equations for translation and rotation:

$$
\begin{gathered}
m \dot{\tilde{\boldsymbol{V}}}=q\left(\boldsymbol{E}+c^{-1} \tilde{\boldsymbol{V}} \times \boldsymbol{B}\right)+\tilde{g} I \nabla \boldsymbol{B} \cdot \boldsymbol{\omega}, \\
I \dot{\boldsymbol{\omega}}=\tilde{g} I \boldsymbol{\omega} \times \boldsymbol{B}-\tilde{g} I \tilde{\boldsymbol{V}} \cdot \nabla \boldsymbol{B} .
\end{gathered}
$$

In these equations, $\nabla$ is the usual 3-vector operator given by $\nabla=\hat{\boldsymbol{e}}^{i} \partial_{i}$. The last term in Equation (32) is the expected force $\nabla \boldsymbol{B} \cdot \boldsymbol{\mu}$ on a magnetic dipole moment in any (nonuniform) magnetic field, time-dependent or not. The second term in Equation (33) is the expected torque $\boldsymbol{\mu} \times \boldsymbol{B}$, while the last term is another torque that is omitted from most textbook presentations. As emphasized by Young [6], that torque must present in order to predict conservation of the total kinetic energy $\frac{1}{2} m \tilde{V}^{2}+\frac{1}{2} I \omega^{2}$ for the case of a nonuniform static magnetic field and zero electric field. During the course of deriving Equations (32) and (33) from the EL equations, the following useful identity must be proved:

$$
\left(a_{b i}^{-1} a_{c j}^{-1}-a_{b j}^{-1} a_{c i}^{-1}\right)\left(\partial a_{k c} / \partial \alpha^{b}\right)=\epsilon_{i j k}
$$

where $\epsilon_{i j k}$ is the Levi-Civita completely antisymmetric three-index symbol. This identity was not trivial to prove. (The author could not find a simple general derivation, and resorted to brute force, calculating and verifying the identity for each symbol, starting from Equation (5) for the matrices $(a)$ and $(a)^{-1}$.) A similar identity exists among the elements of $(b)$. We note that if and only if $\boldsymbol{B}$ has no intrinsic time dependence, one may apply Equation (16) and write Equation (33) as $\dot{\boldsymbol{S}}=\tilde{\boldsymbol{g}} \boldsymbol{S} \times \boldsymbol{B}$, because in such a case $\tilde{\boldsymbol{V}} \cdot \nabla \boldsymbol{B}=\mathrm{d} \boldsymbol{B} / \mathrm{d} t$.

\section{Spin Operators and Statistical Wave Equation}

\subsection{Wave Equation for Arbitrary Spin}

The relevant statistical wave equation for any nonrelativistic system having six coordinates is the six-dimensional version of the general statistical wave Equation (24) of [I], with the unknown constant $\Gamma$ replaced by $\hbar$ :

$$
i \hbar \partial_{t} \psi=\frac{1}{2 m}(-i \hbar \nabla-m u)^{2} \psi+W \psi
$$


Here, the 6-vector field $\boldsymbol{u}$ and the 6-scalar $W$ are the functions that appear in the classical Hamiltonian (31), namely,

$$
\boldsymbol{u}=(q \boldsymbol{A} / m c, \tilde{g} \sqrt{I / m} \boldsymbol{B}), W=(q \varphi, 0),
$$

and the 6-space gradient operator is given by

$$
\nabla=\hat{\boldsymbol{e}}^{i} \partial_{i}+\boldsymbol{e}^{b} \partial_{b},
$$

where $\partial_{b}=\partial / \partial \alpha^{b}$. The Hamiltonian operator in terms of $\boldsymbol{u}$ and $W$ is given by Equation (26) of [I]. However, as shown above, for the model system considered here the classical Hamiltonian may be written as Equation (17) or Equation (31). One need only note that the rotational part of the $6 \mathrm{D}$ gradient operator in Equation (37) is $\boldsymbol{e}^{b} \partial_{b}$, so that in Equation (31) the conjugate classical momenta must be replaced by the momentum operators

$$
\tilde{P}_{i} \rightarrow p_{i}^{o p}=-i \hbar \partial_{i} ; \quad P_{b} \rightarrow p_{b}^{o p}=-i \hbar \partial_{b},
$$

Making these substitutions in Equation (31) yields

$$
H^{o p}=\frac{1}{2 m}\left(p_{i}^{o p}-q c^{-1} A_{i}\right)\left(p_{i}^{o p}-q c^{-1} A_{i}\right)+q \varphi+\frac{1}{2 I}\left(S_{i}^{o p}-I \tilde{g} B_{i}\right)\left(S_{i}^{o p}-I \tilde{g} B_{i}\right),
$$

where, from Equations (29) and (38),

$$
S_{i}^{o p}=a_{b i}^{-1} p_{b}^{o p}=-i \hbar a_{b i}^{-1} \partial_{b} .
$$

Earlier, we showed that despite the torsional character of the Euler angle 3-space, Equation (24) is valid, which ensures that the operators $H^{o p}$ and $S^{o p}$ are Hermitian provided the coordinate probability density obeys periodic boundary conditions w.r. to the Euler angles. It is easy to show that the spin operators $S_{i}^{o p}$ satisfy the usual commutation rules for angular momentum,

$$
\left[S_{i}^{o p}, S_{j}^{o p}\right]=i \hbar \epsilon_{i j k} S_{k}^{o p} \text {. }
$$

One way to obtain this result is to combine Equations (40) and (34). Another way is to write out Equation (40) as a matrix equation, using Equation (5), and then calculate each commutator directly. Equations (29) and (38) also yield the expressions

$$
\bar{S}_{i}^{o p}=b_{b i}^{-1} p_{b}^{o p}=-i \hbar b_{b i}^{-1} \partial_{b} .
$$

Using this equation, and Equation (5) to obtain the matrix (b) and its inverse, one may use Equation (42) directly, or Equation (41) with $\bar{S}_{i}^{o p}=R_{i j} S_{j}^{o p}$, to show that the rotating system spin operators $\bar{S}_{i}^{o p}$ satisfy

$$
\left[\bar{S}_{i}^{o p}, \bar{S}_{j}^{o p}\right]=-i \hbar \epsilon_{i j k} \bar{S}_{k}^{o p} \text {. }
$$

Note the minus sign, compared to Equation (41)! To the best of our knowledge, these left-handed rotating system commutation relations are not mentioned in quantum mechanics textbooks. Evidently, an observer at rest in the coordinate system that rotates with the rigid body would observe this left-handedness in some way. (One would expect that these left-handed rules have been presented in the literature, but we have been unable to locate a 
reference.) After some algebra, either Equation (40) or Equation (42) yields the expression for $\left(\boldsymbol{S}^{o p}\right)^{2}$ in terms of the Euler angles:

$$
\left(\boldsymbol{S}^{o p}\right)^{2}=-\hbar^{2}\left[\partial_{\alpha^{2}}^{2}+\cot \alpha^{2} \partial_{\alpha^{2}}+\left(\sin \alpha^{2}\right)^{-2}\left(\partial_{\alpha^{1}}^{2}+\partial_{\alpha^{3}}^{2}-2 \cos \alpha^{2} \partial_{\alpha^{1}} \partial_{\alpha^{3}}\right)\right] .
$$

The operators $\left(\boldsymbol{S}^{o p}\right)^{2}, S_{3}^{o p}, \bar{S}_{3}^{o p}$ all commute and thus have simultaneous eigenfunctions. These eigenfunctions, sometimes called Wigner harmonics, are proportional to the elements of the matrices of the irreducible representations of the group $\mathrm{SU}(2)$. We shall use Dirac notation and denote them by $\left|s, m_{s}, \bar{m}_{s}\right\rangle$. They satisfy

$$
\begin{gathered}
\left(\boldsymbol{S}^{o p}\right)^{2}\left|s, m_{s}, \bar{m}_{s}\right\rangle=s(s+1) \hbar^{2}\left|s, m_{s}, \bar{m}_{s}\right\rangle, \\
S_{3}^{o p}\left|s, m_{s}, \bar{m}_{s}\right\rangle=m_{s} \hbar\left|s, m_{s}, \bar{m}_{s}\right\rangle, \quad \bar{S}_{3}^{o p}\left|s, m_{s}, \bar{m}_{s}\right\rangle=\bar{m}_{s} \hbar\left|s, m_{s}, \bar{m}_{s}\right\rangle,
\end{gathered}
$$

where $s=0,1 / 2,1,3 / 2,2, \cdots$, and $m_{s}$ and $\bar{m}_{s}$ run independently from $s$ to $-s$ in integer steps. In terms of the Euler angles, all these spin eigenfunctions have the general form [18]

$$
\left|s, m_{s}, \bar{m}_{s}\right\rangle=\exp i\left(m_{s} \alpha^{1}+\bar{m}_{s} \alpha^{3}\right) u_{m_{s}, \bar{m}_{s}}^{s}\left(\alpha^{2}\right),
$$

where the $u_{m_{s}, \bar{m}_{s}}^{s}\left(\alpha^{2}\right)$ can be determined. One can verify this form easily using Equations (40), (45), and (46). We define the raising and lowering operators as follows:

$$
S_{ \pm}^{o p}=S_{1}^{o p} \pm i S_{2}^{o p} ; \bar{S}_{ \pm}^{o p}=\bar{S}_{1}^{o p} \mp i \bar{S}_{2}^{o p} .
$$

Note the change in signs for the rotating frame components. The spin eigenfunctions also satisfy

$$
\begin{aligned}
& S_{ \pm}^{o p}\left|s, m_{s}, \bar{m}_{s}\right\rangle=\hbar \sqrt{\left(s \mp m_{s}\right)\left(s \pm m_{s}+1\right)}\left|s, m_{s} \pm 1, \bar{m}_{s}\right\rangle, \\
& \bar{S}_{ \pm}^{o p}\left|s, m_{s}, \bar{m}_{s}\right\rangle=\hbar \sqrt{\left(s \mp \bar{m}_{s}\right)\left(s \pm \bar{m}_{s}+1\right)}\left|s, m_{s}, \bar{m}_{s} \pm 1\right\rangle,
\end{aligned}
$$

except for phase factors that multiply the square roots but can be set equal to unity with no loss of generality. The relations (45), (46), (47), (49), and (50) may all be derived without reference to Euler angles from the commutation relations (41) and (43) and the physical requirement that the maximum values of $\left|m_{s}\right|$ and $\left|\bar{m}_{s}\right|$ not exceed $s$, see any modern textbook on quantum mechanics, e.g. that by Shankar [19], for the conventional derivation that does not consider the rotating frame contributions. We chose the juxtaposition of signs in the second term of Equation (48) in order that $\bar{S}_{+}^{o p}$ indeed acts to raise the index $\bar{m}_{s}$ by unity, etc.

The statistical/Schrödinger wave equation for the system is

$$
i \hbar \partial_{t} \psi=H^{o p} \psi,
$$

where $H^{o p}$ is given by Equation (39). At first glance, it might seem that the general solution could be written as a superposition of the eigenfunctions $\left|s, m_{s}, \bar{m}_{s}\right\rangle$ over all allowed values of $s$, both integer and half-odd-integer. However, that would violate the boundary condition mentioned above, that all 
observable functions of the Euler angles must be single-valued in intervals of $2 \pi$, i.e., they must be periodic functions with period $2 \pi$, despite the fact that the angles themselves have infinite range. A simple example suffices: Consider a superposition $\psi=C+D \exp i \alpha^{1} / 2$, where $C$ and $D$ are nonzero functions of $x, t$, and the other angles, which is a superposition of $m_{s}=0$ and $m_{s}=1 / 2$ terms. Then the observable probability density $\psi^{*} \psi$ is periodic in $\alpha^{1}$ with period $4 \pi$, not $2 \pi$. Reasoning from this example, it is easy to see that in order to ensure Euler angle periodicities of $2 \pi$ for any probability density and for all other observable functions (which are always bilinear in $\psi^{*}$ and $\psi$ ), the general solution of Equation (51) must be written as a superposition of the spin eigenfunctions with integer $s$ only, or with half-odd-integer $s$ only. (Also, the integer- $s$ eigenfunctions are not orthogonal to the half-odd-integer ones in azimuthal angle inervals $(0,2 \pi)$, which reinforces the above restriction.) Since in this paper we are most interested in the spin-1/2 example, we adopt the superposition of the spin eigenfunctions with half-odd-integer $s$ as the relevant solution of Equation (51). Furthermore, since the $H^{o p}$ for a spherical model particle, Equation (39), contains the $S_{i}^{o p}$ but not the $\bar{S}_{i}^{o p}$, the sum over $\bar{m}_{s}$ is redundant; we may choose any allowed value of $\bar{m}_{s}$ with no loss of generality. Therefore, the relevant general solution of the statistical wave Equation (51) may be written

$$
\psi(x, \alpha, t)=\sum_{s=1 / 2,3 / 2, \cdots m_{s}=-s} \sum_{m_{s}, \bar{m}_{s}}^{s}(x, t)\left|s, m_{s}, \bar{m}_{s}\right\rangle
$$

where any half-odd-integer value of $\bar{m}_{s},-s \leq \bar{m}_{s} \leq s$, may be used. Substitution of Equation (52) in Equation (51) yields $2 s+1$ coupled equations for each $s$. In the next subsection, we write out the equations for $s=1 / 2$.

Before proceeding, we should discuss briefly why the spin eigenfunctions, which have the general form given by Equation (47), are themselves not required to be single-valued in angle intervals $(0,2 \pi)$, in contrast to eigenfunctions like expim $\phi$ involving the spherical polar or cylindrical coordinate azimuthal angle $\phi$. This question has been discussed often; a thoughtful treatment was given by Merzbacher [20]. We paraphrase his answers as follows: The angle $\phi$ helps locate a point in Euclidean 3-space, so values of $\phi$ outside $(0,2 \pi)$ are meaningless: $\phi$ and $\phi+2 n \pi$, with $n$ an integer, are the same points, since e.g. they yield the same Cartesian coordinates for a given choice of the other spherical polar coordinates $(r, \theta)$. Therefore, the eigenfunctions themselves must satisfy periodic boundary conditions in the azimuthal angle interval $(0,2 \pi)$, whereby $m$ must be an integer. (There is some difficulty with this answer in regard to the Aharonov-Bohm effect, which Merzbacher discusses.) In contrast, for the Euler angles, $\alpha^{1}$ and $\alpha^{1}+2 n \pi$ are not the same points, nor are $\alpha^{2}$ and $\alpha^{2}+2 n \pi$, etc., because the object may just keep on rotating, as mentioned above. (Merzbacher points out that the group space of the rotation group is a doubly connected space, whereby the the operation "rotation by $2 \pi$ about an axis" cannot be continuously deformed into the operation "no rotation 


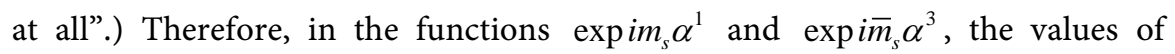
$\left(m_{s}, \bar{m}_{s}\right)$ are restricted to integers or half-odd-integers only by the demands of the spin commutation rules, as discussed above, or, equivalently, by the requirement that all "observable" functions be periodic in Euler angle intervals of $2 \pi$.

There is another consideration pertinent to the discussion just above, as follows. One may always make the change of variables $\xi^{1}=\frac{1}{2}\left(\alpha^{1}+\alpha^{3}\right)$, $\xi^{3}=\frac{1}{2}\left(\alpha^{1}-\alpha^{3}\right)$, e.g., see the treatment of SU(2) by Arfken [15]. Then, for the fundamental intervals $0 \leq\left(\alpha^{1}, \alpha^{3}\right) \leq 2 \pi$, one obtains $0 \leq \xi^{1} \leq 2 \pi,-\pi \leq \xi^{3} \leq \pi$. Furthermore, the eigenfunctions of Equation (47) now involve expi $\left[\left(m_{s}+\bar{m}_{s}\right) \xi^{1}+\left(m_{s}-\bar{m}_{s}\right) \xi^{3}\right]$, which are single-valued in their fundamental coordinate intervals because as discussed above $m_{s}$ and $\bar{m}_{s}$ must both be half-odd-integers, or both integers. This coordinate transformation does not change any of the spin eigenvalues, and it also diagonalizes the metric in the spin space.

Also, it should again be noted that $H^{o p}$ of Equation (39) contains the additional terms $\left(S^{o p}\right)^{2} / 2 I$ and $I(\tilde{g} B)^{2} / 2$ compared to the conventional Hamiltonian operator. In subsection 3.3.3 and the appendix of [I] we analyzed the case of a free rotator, and concluded that a nonrelativistic treatment of hadrons and more massive objects is appropriate, but that a relativistic treatment of leptons is necessary. Such a treatment is beyond the scope of this work.

\subsection{Wave Equation for Spin 1/2}

We examine the solutions of Equation (51) for a given spin angular momentum. For this example, $s=1 / 2$, we may write

$$
\psi_{\bar{m}_{s}}^{s}(x, \alpha, t)=U_{+}(x, t)\left|s,+, \bar{m}_{s}\right\rangle+U_{-}(x, t)\left|s,-, \bar{m}_{s}\right\rangle,
$$

for either allowed value of $\bar{m}_{s}$, as a suitable general solution of Equation (51). Here, $U_{ \pm}$are functions to be determined. For notational convenience, $|s,+,+\rangle$ stands for $\left|\frac{1}{2}, \frac{1}{2}, \bar{m}_{s}\right\rangle$ with $\bar{m}_{s}=1 / 2$, etc. The orthonormal spin eigenfunctions are proportional to the elements of the $2 \times 2$ irreducible representation of $\mathrm{SU}(2)$ in terms of the Euler angles [15]:

$$
\begin{aligned}
& |s,+,+\rangle=(2 \pi)^{-1} \exp \left[i\left(\alpha^{1}+\alpha^{3}\right) / 2\right] \cos \left(\alpha^{2} / 2\right) \equiv u_{++}(\alpha) ; \\
& |s,-,+\rangle=(2 \pi)^{-1} \exp \left[i\left(-\alpha^{1}+\alpha^{3}\right) / 2\right] \sin \left(\alpha^{2} / 2\right) \equiv u_{-+}(\alpha) ; \\
& |s,+,-\rangle=(2 \pi)^{-1} \exp \left[i\left(\alpha^{1}-\alpha^{3}\right) / 2\right] \sin \left(\alpha^{2} / 2\right) \equiv u_{+-}(\alpha) ; \\
& |s,-,-\rangle=(2 \pi)^{-1} \exp \left[-i\left(\alpha^{1}+\alpha^{3}\right) / 2\right] \cos \left(\alpha^{2} / 2\right) \equiv u_{--}(\alpha) .
\end{aligned}
$$

It is very simple to verify that these eigenfunctions are indeed orthonormal under integration over the spin space (rotation group) volume, e.g., that 


$$
\langle s,+,+\mid s,+,+\rangle=\int_{0}^{2 \pi} \mathrm{d} \alpha^{1} \int_{0}^{2 \pi} \mathrm{d} \alpha^{3} \int_{0}^{\pi} \mathrm{d} \alpha^{2} \sin \alpha^{2} u_{++}^{*} u_{++}=1,
$$

etc. Using Equations (40), (42), and (44), it is also straightforward to verify that these functions satisfy Equations (45), (46), (49), and (50) for $s=1 / 2$.

We write the Hamiltonian operator (39) as

$$
H^{o p}=H_{1}^{o p}+H_{2}^{o p},
$$

where

$$
\begin{aligned}
& H_{1}^{o p}=\frac{1}{2 m}\left(\boldsymbol{p}^{o p}-q \boldsymbol{A} / c\right)^{2}+q \varphi+\frac{1}{2 I}\left(\boldsymbol{S}^{o p}\right)^{2}+\frac{1}{2} I \tilde{g}^{2} B^{2} ; \\
& H_{2}^{o p}=-\tilde{g} \boldsymbol{B} \cdot \boldsymbol{S}^{o p}=-\tilde{g}\left[B_{3} S_{3}^{o p}+\frac{1}{2}\left(B_{-} S_{+}^{o p}+B_{+} S_{-}^{o p}\right)\right]
\end{aligned}
$$

Here, $S_{ \pm}^{o p}$ are given by Equation (48), and $B_{ \pm}=B_{1} \pm i B_{2}$. Since we may use either value of $\bar{m}_{s}$, we choose $+1 / 2$. Then a little algebra, using Equations (45), (46), (49), (50), (51), and (53)-(55), yields two coupled equations,

$$
\begin{aligned}
& \left(-i \hbar \partial_{t}+H_{1}^{o p}\right) U_{+}-\frac{1}{2} \hbar \tilde{g}\left(B_{3} U_{+}+B_{-} U_{-}\right)=0 ; \\
& \left(-i \hbar \partial_{t}+H_{1}^{o p}\right) U_{-}+\frac{1}{2} \hbar \tilde{g}\left(B_{3} U_{-}-B_{+} U_{+}\right)=0 .
\end{aligned}
$$

Of course, these two coupled equations may be written as a $2 \times 2$ matrix equation. If one defines the matrices

$$
\sigma_{1}=\left(\begin{array}{cc}
0 & 1 \\
1 & 0
\end{array}\right) ; \quad \sigma_{2}=\left(\begin{array}{cc}
0 & -i \\
i & 0
\end{array}\right) ; \quad \sigma_{3}=\left(\begin{array}{cc}
1 & 0 \\
0 & -1
\end{array}\right),
$$

which happen to be the Pauli spin matrices, and also defines the column matrix (spinor)

$$
(U)=\left(\begin{array}{c}
U_{+} \\
U_{-}
\end{array}\right) \text {, }
$$

and applies Equation (58), then one immediately obtains the matrix equation

$$
\left(-i \hbar \partial_{t}+H_{1}^{o p}\right)(U)-\left(\tilde{g} B_{i}\right)\left(\frac{1}{2} \hbar \sigma_{i}\right)(U)=0 .
$$

This is the Pauli-Schrödinger equation, except that $H_{1}^{o p}$ contains the additional terms $\frac{1}{2 I}\left(\boldsymbol{S}^{o p}\right)^{2}+\frac{1}{2} I \tilde{g}^{2} B^{2}$, which must be present for the reasons discussed above. Also, this equation is valid for any value of $g$ in the magnetogyric ratio $\tilde{g}=g q / 2 m c$, not just for the value $g=2$ originally chosen for the electron in order to match atomic spectral data. Note that as usual the definition $\tilde{S}_{i}^{o p}=\frac{1}{2} \hbar \sigma_{i}$ yields the conventional matrix representation of the non-rotating frame Cartesian components $S_{i}$ of the spin angular momentum operator. Also, we remark again that exactly the same Equations (58) and (61) result if we use the spin eigenfunctions for $\bar{m}_{s}=-1 / 2$ instead of those for $+1 / 2$. It is also noteworthy that the "magic" factorization 
$\left(\boldsymbol{p}^{o p}-q \boldsymbol{A} / c\right)^{2} \rightarrow\left[\boldsymbol{\sigma} \cdot\left(\boldsymbol{p}^{o p}-q \boldsymbol{A} / c\right)\right]^{2}$ of the translational Hamiltonian (see e.g. Sakurai [21]), the nonrelativistic analog of the Dirac factorization, allows only $g=2$ and also does not provide the terms $\frac{1}{2 I}\left(S^{o p}\right)^{2}+\frac{1}{2} I \tilde{g}^{2} B^{2}$. Since $g=2$ is correct for the electron without radiative corrections, and since that factor arises from the factorization of the translational Hamiltonian, perhaps the electron spin actually originates from translational zitterbewegung induced by the SZPF, as has been proposed [8] [10]. After all, as implied by the discussion to follow and the material in the appendix, it is not really clear how to distinguish spin from orbital angular momentum in models of rotating rigid bodies.

\section{Summary and Discussion}

\subsection{Summary}

This work concerned a system of one rigid object having a mass distribution with nonzero total mass, an electric charge distribution, and intrinsic or "spin" angular momentum and associated magnetic moment, which for nonrelativistic motions requires a six-dimensional metric space for three CM coordinates and three Euler angle coordinates. In Section 2 we followed the development by R. Young [6] and showed that the magnetic field appears in the classical nonrelativistic Hamiltonian as a gauge field associated with the space-fixed frame Cartesian components of the spin angular momentum conjugate to the Euler angles. In Section 3, we applied the new general rules derived in Section 2 of [I] to obtain the six-dimensional CST Schrödinger equation using the classical Hamiltonian. We provided a new proof that the non-rotating CM frame Cartesian components of the canonical spin angular momentum become operators that are linear combinations of derivatives with respect to the Euler angles and obey the conventional commutation rules for quantum angular momentum operators. We also provided a proof of a result that apparently is not mentioned in textbooks, that the co-rotating-frame Cartesian components of the spin operator obey left-handed commutation rules. Furthermore, we showed that a particle wavefunction may be a superposition of half-odd-integer or integer spin eigenstates, but not both. This correct result follows in a new way, from applying physical boundary conditions in the Euler angle description. For a particular spin $s$, we showed that the general six-dimensional Schrdinger equation yields $2 s+1$ coupled equations for the amplitudes $\psi_{m_{s}, \bar{m}_{s}}^{s}(x, t)$ in Equation (52). We also showed that, for spin $1 / 2$, these coupled equations reduce to the Pauli-Schrdinger equation, with the usual Pauli matrix representation of the space-fixed system spin operators and with two-component spinors, but with arbitrary magnetogyric ratio and additional rigid rotator terms in the Hamiltonian. In Appendix A, we showed that the usual spin commutation rules apply to a rigid rotator of any structure, not just to a spherically symmetric rotator. This new derivation yields the well-known fact that any object in a rigid rotator eigenmode must have spin equal to one of the eigenvalues 
$s=(0,1 / 2,1,3 / 2, \cdots)$, regardless of its internal structure or how many subparticles it contains, etc. However, the rotational Hamiltonian and its eigenfunctions and eigenvalues do depend on the particle structure.

\subsection{Discussion}

One topic that seems to merit discussion is the distinction between spin and orbital angular momentum. The distinction is fuzzy at best. For example, consider the classical model of an extended object as a cloud containing many point particles; see e.g. Goldstein's textbook [14]. The total angular momentum of the cloud may always be defined with respect to an arbitrarily chosen origin of coordinates, and it may always be written (nonrelativistically) as the orbital angular momentum of the total mass, located at the CM, as it moves about the origin, plus the sum of the orbital angular momenta of each of the constituent point particles about the CM. So in general all the angular momentum in such a model is orbital. The total orbital angular momentum about the CM is called the intrinsic angular momentum of the object. If the point particles in the cloud are rigidly bound to each other, so that all their motions relative to the $\mathrm{CM}$ can be represented in terms of a single angular velocity vector that requires only three Euler angle coordinates to describe, then that intrinsic orbital angular momentum is called the (classical) spin angular momentum. Given the above, how can we justify allowing half-odd-integer spin angular momentum, but only integer orbital angular momentum? One response, adopted by many, is that we cannot justify it, so we simply go with the predictions of the commutation rules, to be used as needed. Another possible response is to note that the Goldstein model may not be the appropriate one. In actuality, all collections of particles and thus all "rigid" bodies are comprised of electrons, quarks, (and photons and gluons and...), as far as we know. The fermions and bosons in this mix have their own irreducible spins, analogously to subvortices within larger vortices in fluid mechanics, not the case for the subparticles in the Goldstein model. Thus in nonrelativistic theory it would seem that only in a model utilizing fundamental subparticles having irreducible spins can one hope to distinguish between orbital and spin angular momentum. So this argument turns into an argument for the existence of such fundamental particles. (In relativistic field theory, the distinction between orbital and spin angular momentum follows from Noether's theorem; see e.g. the paper by the author on stress-energy tensors [22].)

Another topic that should be mentioned is the CST method for treating systems of more than one particle. As discussed in [I] in some detail, the CST and QM methods are mathematically the same. The SWE for a nonrelativistic system of several identical or different particles is the same as the SEQ for the system. The CST for nonrelativistic systems of arbitrarily many identical particles is the same mathematically as quantum many-body theory for those particles. For example, for two identical or different nonrelativistic rigid rotators, one needs three CM coordinates and three Euler angles for each rotator, so the 
configuration space of the SWE/SEQ is altogether 12-dimensional with a block-diagonal metric.

\section{Conclusions}

The classical statistical theory (CST) and its statistical wave equation (SWE) developed in [I] must apply to any nonrelativistic classical Lagrangian system. Furthermore, the SWE is in general the same as the Schrdinger equation (SEQ) for the system, and has the same sets of solutions, once an unknown constant is set equal to $\hbar$. The SWE for a classical model charged rigid rotator having spherical or cylindrical symmetry or arbitrary morphology is not exactly the same as the conventional SEQ for the system; the differences occur because the conventional SEQ incorrectly omits terms from the Hamiltonian when an external magnetic field is present (see Section 3.2 above, and Appendix A). Nevertheless, the spin operators and their eigenfunctions and eigenvalues are exactly the same for the SWE and SEQ.

On the basis of the general results in [I] and the results for the important example treated in this paper, it is tempting to conclude that the CST should replace conventional quantum mechanics, at least for nonrelativistic systems. However, as discussed in [I], the identical solutions of the SWE/SEQ must be interpreted differently, and the equation provides only an incomplete description of the statistics of such systems, albeit one that must be obeyed. Also, after nearly a century, it should require a great deal of evidence and thought to reverse the current points of view that coordinate trajectories do not exist and quantized energies do. Thus, as stated in [I], we still feel that it is premature to reach the abovementioned conclusion. In particular, a similar CST for relativistic motions of general many-particle systems should be found before such a conclusion might be justified.

\section{References}

[1] Goedecke, G. (2017) Journal of Modern Physics, 8, 786-807. https://doi.org/10.4236/jmp.2017.85050

[2] Bopp, F. and Haag, R. (1950) Zeitschrift für Naturforschung, 5a, 644.

[3] Nyborg, P. (1962) Nuovo Cimento, 23, 47. https://doi.org/10.1007/BF02733541

[4] Corben, H.C. (1968) Classical and Quantum Theories of Spinning Particles. Holden-Day, San Francisco.

[5] De la Peña-Auerbach, L. (1971) Journal of Mathematical Physics, 12, 453. https://doi.org/10.1063/1.1665609

[6] Young, R. (1976) American Journal of Physics, 44, 581. https://doi.org/10.1119/1.10383

[7] Barut, A. and Bracken, A. (1981) Physical Review D, 23, 2454. https://doi.org/10.1103/PhysRevD.23.2454

[8] Barut, A. and Bracken, A. (1981) Physical Review D, 24, 3333. https://doi.org/10.1103/PhysRevD.24.3333

[9] Jáuregi, R. and de la Peña, L. (1981) Physics Letters A, 86, 280. 
[10] Barut, A. and Zanghi, N. (1984) Physical Review Letters, 52, 2009. https://doi.org/10.1103/PhysRevLett.52.2009

[11] Barut, A., Božić, M. and Marić, Z. (1992) Annals of Physics, 214, 53.

[12] Arsenovic, D., Barut, A., Marić, Z. and Božić, M. (1995) Nuovo Cimento B, 110, 163. https://doi.org/10.1007/BF02741499

[13] Griffiths, D. (2005) Introduction to Quantum Mechanics. 2nd Edition, Prentice-Hall, London.

[14] Goldstein, H. (1980) Classical Mechanics. 2nd Edition, Addison-Wesley, Reading.

[15] Arfken, G. (1985) Mathematical Methods for Physicists. 3rd Edition, Academic Press.

[16] Lichnerowicz, A. (1962) Elements of Tensor Calculus. Methuen, London.

[17] Hartle, J. (2003) Gravity. Addison-Wesley, Reading.

[18] Mathews, J. and Walker, R. (1970) Mathematical Methods of Physics. 2nd Edition, W. A. Benjamin, New York.

[19] Shankar, R. (1994) Principles of Quantum Mechanics. 2nd Edition, Springer, New York. https://doi.org/10.1007/978-1-4757-0576-8

[20] Merzbacher, E. (1962) American Journal of Physics, 30, 237. https://doi.org/10.1119/1.1941984

[21] Sakurai, J. (1967) Advanced Quantum Mechanics. Addison-Wesley, Reading.

[22] Goedecke, G. (1974) Journal of Mathematical Physics, 15, 72. 


\section{Appendix A: Arbitrary Nonrelativistic Rigid Rotator}

In this appendix we first show that the spin operators and their commutation rules for a nonrelativistically rigidly rotating object of arbitrary structure are the same as for a spherically symmetric object. Then we obtain the Hamiltonian of the former interacting with a magnetic field.

\subsection{Spin Commutation Rules}

We make use of the fact that one may express the inertia tensor of any object in a body-fixed principal axis Cartesian frame in which the tensor is diagonal with principal moments of inertia $\bar{I}_{i}, i=1,2,3$. The rotational kinetic energy is

$$
T_{\text {rot }}=\frac{1}{2} \bar{I}_{i} \bar{\omega}_{i}^{2}=\frac{1}{2} m g_{b c} \dot{\alpha}^{b} \dot{\alpha}^{c},
$$

where the middle term extends the summation convention to indices repeated twice, which is notationally convenient. Applying Equation (4) of the text yields

$$
g_{b c}=m^{-1} \overline{I_{i}} b_{i b} b_{i c} \text {. }
$$

Here, $m$ is a parameter having dimension mass that may be chosen as the mass of the object, $g_{b c}$ is the covariant metric in the 3-space of the Euler angles $\alpha^{b}$, and the $b_{i b}$ are given by Equation (5). It is easy to see that the covariant and contravariant basis vectors in the Euler angle space are given by

$$
\boldsymbol{e}_{b}=\left(\bar{I}_{i} / m\right)^{1 / 2} b_{i b} \hat{\overline{\boldsymbol{e}}}_{i} ; \quad \boldsymbol{e}^{b}=\left(m / \bar{I}_{i}\right)^{1 / 2} b_{b i}^{-1} \hat{\hat{\boldsymbol{e}}_{i}},
$$

where the $\hat{\overline{\boldsymbol{e}}}_{i}$ are the Cartesian unit basis vectors in the body-fixed frame. These relations are the analogs of Equations (20) and (21). Then

$$
g^{b c}=\boldsymbol{e}^{b} \cdot \boldsymbol{e}^{c}=m\left(\bar{I}_{i}\right)^{-1} b_{b i}^{-1} b_{c i}^{-1} .
$$

It is also easy to see that $\boldsymbol{e}_{b} \cdot \boldsymbol{e}^{c}=\delta_{b}^{c}$ and that $g^{b c} g_{c a}=\delta_{a}^{b}$, as must be the case. Now consider a freely rotating particle, so that its rotational Lagrangian $L_{r o t}=T_{\text {rot }}$. Than the conjugate (angular) momenta are $P_{b}=\partial L_{r o t} / \partial \dot{\alpha}^{b}=m g_{b c} \dot{\alpha}^{c}$, whereby

$$
\dot{\alpha}^{b}=m^{-1} g^{b c} P_{c} .
$$

Using Equations (4), (65), and (66), one obtains

$$
\bar{S}_{1}=\bar{I}_{1} \bar{\omega}_{1}=b_{c 1}^{-1} P_{c},
$$

and similarly for the other spin angular momentum components in the body-fixed frame. Then, with $P_{c} \rightarrow p_{c}^{o p}=-i \hbar \partial / \partial \alpha^{c}$ as in Equation (38), one obtains

$$
\bar{S}_{i} \rightarrow \bar{S}_{i}^{o p}=-i \hbar b_{c i}^{-1} \partial / \partial \alpha^{c} .
$$

These operators are identical to those defined by Equation (42), which means that they are independent of the structure of the rigidly rotating particle. Furthermore, since $b_{i b}=R_{i j} a_{j b}$ and $\bar{S}_{i}^{o p}=R_{i j} S_{j}^{o p}$, one also obtains

$$
S_{i}^{o p}=-i \hbar a_{c i}^{-1} \partial / \partial \alpha^{c},
$$


the same as Equation (40). Therefore, the commutation relations of Equations (41) and (43) are still valid, and the simultaneous eigenfunctions of $\left(\boldsymbol{S}^{o p}\right)^{2}, S_{3}^{o p}, \bar{S}_{3}^{o p}$ are still the $\left|s, m_{s}, \bar{m}_{s}\right\rangle$ that satisfy Equation (45), (46), (49), and (50). However, the free particle Hamiltonian operator is easily seen to be

$$
H_{\text {rot }}^{o p}=\frac{1}{2} \bar{I}_{i}^{-1} \bar{S}_{i}^{o p} \bar{S}_{i}^{o p}
$$

If all three principal moments of inertia are different, the individual spin eigenfunctions are not eigenfunctions of $H_{\text {rot }}^{o p}$. However, suppose the object has a rotational symmetry axis, which we may always choose to be the body-fixed $z$-axis, so that $\bar{I}_{1}=\bar{I}_{2} \neq \bar{I}_{3}$. Then

$$
H_{\text {rot }}^{o p}=\frac{1}{2}\left[\bar{I}_{1}^{-1} \bar{S}_{i}^{o p} \bar{S}_{i}^{o p}+\left(\bar{I}_{3}^{-1}-\bar{I}_{1}^{-1}\right) \bar{S}_{3}^{o p} \bar{S}_{3}^{o p}\right] \text {. }
$$

The individual spin eigenfunctions $\left|s, m_{s}, \bar{m}_{s}\right\rangle$ are eigenfunctions of this Hamiltonian; the eigenvalues are

$$
E_{s, \bar{m}_{s}}=\frac{1}{2}\left[\bar{I}_{1}^{-1} s(s+1) \hbar^{2}+\left(\bar{I}_{3}^{-1}-\bar{I}_{1}^{-1}\right) \bar{m}_{s}^{2} \hbar^{2}\right] .
$$

This result reveals that some of the degeneracy of the spherical rigid rotator Hamiltonian eigenvalues for a given $s$ may be removed for an axially but not spherically symmetric object, with the energy shifts dependent on the quantum number $\bar{m}_{s}$ associated with the body-fixed frame.

We emphasize again that the spin operators, their commutation relations, their eigenfunctions and eigenvalues, and the raising and lowering operator relations (50) and (51) are independent of the principal moments of inertia and any other model particle parameters. This result is not surprising in view of the fact that the rotation of any rigid object is described in terms of three angles that simply relate the time-dependent orientation of a rotating orthonormal triad of basis vectors relative to a nonrotating orthonormal triad. The result implies that any object must have half-odd-integer or integer spin when it is in a rigid rotation mode, regardless of how many subparticles it contains and how they are distributed, which does seem to be the case for baryons, nucleons, atoms, molecules, etc. For example, the three quarks in a nucleon are now thought to have both spin angular momentum and orbital angular momentum about the nucleon center of momentum, and the gluons may contribute to the total angular momentum as well, but all that internal structure must arrange itself so that the total spin of a nucleon in its ground state is $1 / 2$.

\subsection{Interaction with a Magnetic Field}

Consider a rigidly rotating charged or uncharged extended particle of arbitrary structure. The Cartesian components of the particle's intrinsic magnetic moment in the rotating system are expressible as linear functions of the rotating system angular velocity components:

$$
\bar{\mu}_{i}=\bar{Q}_{i j} \bar{\omega}_{j},
$$


where $\bar{Q}_{i j}=\bar{Q}_{j i}$ are constants, components of a symmetric rank two tensor that could be obtained from the definition of Equation (7) with $f_{q}=f_{q}\left(x^{\prime}\right)$, not spherically symmteric, for the magnetic moment of a given current density $\boldsymbol{J}$. Then for a particle with its CM position $\boldsymbol{X}$ at rest, the relevant Lagrangian is

$$
L_{\text {rot }}=\frac{1}{2} \bar{I}_{i} \bar{\omega}_{i} \bar{\omega}_{i}+\bar{Q}_{i j} \bar{\omega}_{i} \bar{B}_{j}
$$

where $\bar{B}_{j}=R_{j k} B_{k}$ are defined just after Equation (25); they are effective rotating frame Cartesian components of any magnetic field that may be present. These components are evaluated at $\boldsymbol{X}$, and they also depend on the Euler angles. The spin angular momentum conjugate to $\bar{\omega}_{i}$ is

$$
\bar{S}_{i}=\partial L_{\text {rot }} / \partial \bar{\omega}_{i}=\bar{I}_{i} \bar{\omega}_{i}+\bar{Q}_{i j} \bar{B}_{j},
$$

which from the definition $H_{\text {rot }}=\bar{\omega}_{i} \bar{S}_{i}-L_{\text {rot }}$ yields the rotational Hamiltonian

$$
H_{\text {rot }}=\frac{1}{2} \bar{I}_{i}^{-1}\left(\bar{S}_{i}-\bar{Q}_{i k} \bar{B}_{k}\right)\left(\bar{S}_{i}-\bar{Q}_{i l} \bar{B}_{l}\right)
$$

The cross-term in the quadratic form above is the apparent interaction Hamiltonian

$$
H_{\text {int }}=-\frac{1}{2} \bar{I}_{i}^{-1} \bar{Q}_{i k}\left(\bar{B}_{k} \bar{S}_{i}+\bar{S}_{i} \bar{B}_{k}\right) .
$$

Clearly, this interaction is a more complicated form than in the spherically symmetric case, and we cannot go further than Equation (A16) in the case of arbitrary particle structure. For illustration, consider the special case in which $\bar{Q}_{i k}=\delta_{i k} \bar{Q}_{i}$, so that the prinipal axis coordinate system for the charge distribution is the same as that for the mass distribution. Also, let the ratio

$$
\bar{Q}_{i} / \bar{I}_{i}=\tilde{g}
$$

not summed over $i$, be the same for each principal axis, where $\tilde{g}=g q / 2 m c$ as in Equation (13) for a particle of charge $q$, but is to be chosen or eveluated using appropriate integrals for an uncharged particle. Then

$$
H_{\text {int }}=-\frac{1}{2} \tilde{g}\left(\bar{B}_{i} \bar{S}_{i}+\bar{S}_{i} \bar{B}_{i}\right)
$$

When $\bar{S}_{i}$ is replaced by $\bar{S}_{i}^{o p}$, it is not algebraically trivial to show that the commutator $\left[\bar{S}_{i}^{o p}, \bar{B}_{i}\right]=0$. The proof reduces to showing that $R_{i j}\left(S_{j}^{o p} R_{j k}\right)=0$, which can be done using Equation (A8) and several equations in the text, or by starting with the definition of the 3-vector angular velocity, Equation (3). Therefore, in this quite special but still not spherically symmetric case, the effective interaction Hamiltonian is the same as for a spherically symmetric particle, namely, $H_{\text {int }}=-\tilde{g} \bar{B}_{i} \bar{S}_{i}=-\tilde{g} B_{i} S_{i}$. Therefore, for spin $s$, one still obtains $2 s+1$ coupled wave equations; for $s=1 / 2$, these equations still yield the Pauli matrices, but the free particle part of the Hamiltonian operator is given by Equation (A10) for the case of an object having cylindrical but not spherical symmetry.

As mentioned in the text after Equation (12), a simple $g$-factor exists and is 
exactly equal to 2 for a circular disk rotator having mass uniformly distributed in the disk and charge uniformly distributed around the circumference. In order to derive this result for a disk of radius $a$, we first note that, in the principal axis cylindrical coordinate system $(r, \phi, z)$, the normalized mass and charge distribution functions $f_{m}, f_{q}$ are given by $f_{m}=\left(1 / \pi a^{2}\right) \Theta(a-r) \delta(z)$ and $f_{q}=(1 / 2 \pi a) \delta(r-a) \delta(z)$, where $\Theta$ is the unit step function, equal to unity for positive argument and zero for negative argument, and $\delta$ is the Dirac delta. Utilizing Equations (6) and (9) with these nonspherical distributions easily yields the following results for the rotating (barred) system components of the magnetic moment and the kinetic spin angular momentum:

$$
\begin{gathered}
\bar{\mu}_{z}=\left(q a^{2} / 2 c\right) \bar{\omega}_{z} ; \quad \bar{S}_{z}^{K}=\left(m a^{2} / 2\right) \bar{\omega}_{z} ; \\
\bar{\mu}_{x, y}=\left(q a^{2} / 4 c\right) \bar{\omega}_{x, y} ; \quad \bar{S}_{x, y}^{K}=\left(m a^{2} / 4\right) \bar{\omega}_{x, y} ;
\end{gathered}
$$

Therefore, we have $\bar{\mu}_{i}=2(q / 2 m c) \bar{S}_{i}^{K}$, the same for every rotating frame Cartesian component, so we may write $\mu=2(q / 2 m c) S^{K}$, whereby $g$ exists and is equal to 2 for this model rotator. 